\title{
Deletion of an Enhancer in FGF5 is Associated With Ectopic Expression in Goat Hair Follicles and the Cashmere Growth Phenotype
}

\section{Yefang Li}

Chinese Academy of Agricultural Sciences Institute of Animal Science https://orcid.org/0000-00034010-734X

\section{Shen Song}

Chinese Academy of Agricultural Sciences Institute of Animal Science

Xuexue Liu

Chinese Academy of Agricultural Sciences Institute of Animal Science

\section{Yanli Zhang}

Chinese Academy of Agricultural Sciences Institute of Animal Science

\section{Dandan Wang}

Chinese Academy of Agricultural Sciences Institute of Animal Science

\section{Xiaohong He}

Chinese Academy of Agricultural Sciences Institute of Animal Science

\section{Qianjun Zhao}

Chinese Academy of Agricultural Sciences Institute of Animal Science

\section{Yabin Pu}

Chinese Academy of Agricultural Sciences Institute of Animal Science

\section{Weijun Guan}

Chinese Academy of Agricultural Sciences Institute of Animal Science

\section{Yuehui Ma}

Chinese Academy of Agricultural Sciences Institute of Animal Science

Lin Jiang ( $\nabla$ jianglin@caas.cn )

Chinese Academy of Agricultural Sciences Institute of Animal Science

\section{Research}

Keywords: Cashmere growth, Whole-gonome sequencing, FGF5, Indel, Enhancer

Posted Date: October 2nd, 2020

DOI: https://doi.org/10.21203/rs.3.rs-84328/v1 
License: (c) (i) This work is licensed under a Creative Commons Attribution 4.0 International License. Read Full License 


\section{Abstract}

\section{Background}

Research on cashmere growth has a significant effect on the production of cashmere and a profound influence on cashmere goat breeding. Whole-genome sequencing is a powerful platform to rapidly gain novel insights into the identification of genetic mechanisms underlying cashmere fiber growth.

\section{Results}

In this study, we generated whole-genome sequences of 115 domestic goats from China, Nepal and Pakistan, including 51 cashmere goats and 64 non-cashmere goats. We found genetically distinct clusters according to their geographic locations but genetic admixture or introgression may have occurred between the Chinese and Nepalese goats. We identified that the fibroblast growth factor 5 gene (FGF5) shows a strong signature for positive selection in the cashmere goat. The 505-bp indel variant at the FGF5 gene locus appeared to be strongly associated with cashmere growth. Functional validation showed that the insertion variant may serve as an enhancer for transcription factor binding, resulting in increased transcription of the upstream FGF5 gene in non-cashmere goats.

\section{Conclusions}

Our study provides useful information for the sustainable utilization and improved conservation of goat genetic resources and demonstrates that the indel mutation in the FGF5 gene could potentially serve as a molecular marker of cashmere growth in cashmere goat breeding.

\section{Background}

Cashmere wool, usually simply known as cashmere, is a fiber obtained from cashmere goats, pashmina goats, and some other breeds of goat. This fiber has been used to make yarn, textiles and clothing for hundreds of years. Cashmere is closely associated with the Kashmir shawl; the word "cashmere" is derived from an anglicization of Kashmir, which occurred in the 19th century when the Kashmir shawl reached Europe from Colonial India [1]. Common usage defines the fiber as wool, but it is finer, stronger, lighter, softer and approximately three times more insulating than sheep wool [2].

Cashmere has been manufactured in China, Mongolia, Nepal and Pakistan for thousands of years. China has the largest number and richest variety of cashmere goats, such as the Inner Mongolia cashmere, Liaoning and Tibetan varieties, and has become the largest producer of raw cashmere, estimated at 15,438 metric tons (in hair) per year [3]. Nepal has a sizeable indigenous goat population with many nondescript goats. Nepalese goat breeds exhibit enormous variations in fecundity; meat, milk and fibre production; disease resilience; and nutritional requirements. Pakistan is the fourth largest goat-producing country after China, India and Nigeria (FAOSTAT, http://www.fao.org/faostat). The major purposes of 
Pakistani goats are milk, meat and hair [4]. Moreover, some studies have suggested that a second domestication event for cashmere breeds took place in Pakistan [5].

Cashmere goats grow a double coat composed of the guard hair produced by primary hair follicles (PHFs) and the cashmere produced by the secondary hair follicles (SHFs) $[6,7]$. The staple length and diameter of hair fibers are the main indicators used to evaluate the value of cashmere. Therefore, identification of related genes and molecular mechanisms that regulate cashmere traits is of great significance. In recent years, based on the goat reference genome, several studies have attempted to characterize genetic variations of cashmere fiber traits in different goat populations using a wholegenome sequencing strategy. For instance, the genes PRDM6, FGF5 [8], LHX2, FGF9, WNT2 [9], SGK3, IGFBP7, OXTR [10], and so on, showed a strong selection signature for cashmere growth and length in Chinese goat populations. However, to our knowledge, few studies have identified the causative mutations of the goat FGF5 gene that underlie cashmere growth in goats. Moreover, the sample size in cashmere goat studies has been limited to Chinese goat breeds, which may not comprehensively analyze cashmere traits.

Here, we sequenced the whole genomes of 115 goats representing 15 breeds from habitats in China, Nepal and Pakistan. To identify the genetic basis for cashmere growth trait in cashmere goats, we performed genomic analysis of selection signatures of goats and identified that the FGF5 gene showed some of the strongest signatures for positive selection in the cashmere goat genome. Further exploration of the FGF5 genotypes and functional validation assays indicated that a 505-bp indel mutation located downstream from FGF5 gene may act as an enhancer, resulting in increased mRNA expression of the FGF5 gene; moreover, deletion of this enhancer is strongly associated with cashmere growth in cashmere goats.

\section{Methods}

\section{Animal ethics}

All sample collection were approved by the Animal Welfare and ethics Committee of Institute of Animal Science, Chinese Academy of Agricultural Sciences (Approval number: IAS2019-61).

\section{Sample information}

In this study, we collected a total of 91 goats representing 44 Chinese native goats, 16 Nepalese goats and 31 Pakistani goats for whole-genome sequencing. In addition, we downloaded genomic dataset of 24 Chinese goats from the Sequencing Read Archieve (https://www.ncbi.nlm.nih.gov/) under accession code PRJNA338022. 51 of the total 115 goats are cashmere goats, including 8 Liaoning (LiNi), 6 Arbus (ABS), 7 Erlangshan (ELS), 3 Alashan (ALS), 10 Tibetan Bange (TBG), 10 Tibetan Ritu (TRT) in China and 7 Nepalese highland (NPH) in Nepal. 64 native goats produce little cashmere, including 10 Toggenburg dairy (TGB), 7 Laoshan dairy (LaSh), 7 Xiangdong black (XiDo) in China, 9 Nepalese lowland (NPL) in Nepal, 6 Bugi Toori (BTR), 9 Kamori (KMR), 11 Pateri (PTR) and 5 Tapri (TPR) in Pakistan (Table S1, Fig 
1A). A minimum of two separate flocks were sampled for each breed or location, and parent/offspring pairs were excluded.

\section{Whole genome sequencing analysis}

DNA extraction was conducted by Wizard® Genomic DNA Purification Kit (Promega). About $3 \mu \mathrm{g}$ of genomic DNA from each collected sample was sequenced on Illumina HiSeq 2000 instruments at BerryGenomics Company (Beijing, China). The 350bp sequencing library with paired-end sequencing was constructed using Illumina's standard protocol. At least $5 x$ genome coverage and no less than $15 \mathrm{~Gb}$ sequencing data were gained per individual. After trimming low-quality bases and adapter sequences, the clean reads were aligned against the latest goat reference genome assembly ARS1(GCF_001704415.1) [11] using mem algorithm in Burrows-Wheeler Aligner (BWA) software [12,13]. Then the mapping results were converted to BAM format by SAMtools (Version: 1.1) [14]and sorted by SortSam tools in Picard packages (picard.sourceforge.net, Version: 1.86). Only properly paired reads both aligned to the reference were retained for subsequent analysis (Table S2). The BamCoverage (https://github.com/BGIshenzhen/BamCoverage/) was used to compute the coverage and depth of sequence alignments, with the "statistics Coverage" parameter.

\section{Variant calling}

The program Genome Analysis Toolkit (GATK) [15]and SAMtools v0.1.19 [16] was used to identify SNPs, short insertions and deletions (indels). Reads were realigned around indels using the Realigner Target Creator and Indel Realigner tools from GATK, before calling SNPs with the GATK Unified Genotype and SAMtools mpileup modules, separately. SNPs were retained if matching the following five criteria: (1) the SNP confidence score (QD) was greater than or equal to 20(2) the Phred-scaled P-value of the Fisher's exact test to detect strand bias (FS) was inferior to equal to 10; (3) the Z-score of the Wilcoxon rank sum test of Alt vs. Ref read position bias (ReadPosRankSum) was greater or equal to -8; (4) the Qualscore of each individual SNP was larger-than-average; (5) SNPs showed only two possible alleles and a minimal allele frequency of $5 \%$. The variants with sequence coverage and base-level values lower than the average of all sites were filtered. The 115 individual SNP VCF files were combined into the merged dataset of 17,534,538 autosomal SNPs and this merged SNP dataset was further phased to impute its own missing positions using BEAGLE software $[17,18]$.

\section{Annotation}

SNP variants were classified into protein coding regions (overlapping a coding exon), 5'UTRs and 3'UTRs (overlapping untranslated region), intronic regions (overlapping with an intron), or intergenic regions using the goat genome GTF file downloaded from Ensembl 94 (ftp://ftp.ensembl.org/pub/release100/gtf/capra_hircus/) and the SNPEff software (Version: 4.0) [19]. SNPs located within protein coding regions were further binned into synonymous and non-synonymous SNPs (Table S3).

\section{Diversity analysis}


The within-population genetic diversity for goat populations was assessed using the filtered SNPs and various metrics, including observed $(\mathrm{Ho})$ and expected heterozygosity $(\mathrm{He})$ (Table S4). The runs of homozygosity (ROH) for each goat population, including the number of ROHs and the total size within ROHs for each individual, were calculated by the command of "-homozyg-window-snp 50 -homozygwindow-het 1 -homozyg-kb 500 -homozyg-density 1000" using the program PLINK v1.90b [20]. Linkage disequilibrium (LD) was computed for each population via the squared correlation coefficient $\left(r^{2}\right)$ between pairwise SNPs by the command of "-MaxDist 500 -MAF 0.005 -Het 0.9 -Miss 0.25 " using the software PopLDdecay (https://github.com/BGI-shenzhen/PopLDdecay)

\section{Phylogenetic analysis}

All SNPs were pruned using PLINK (Version:1.90b) and considering window sizes of 1000 variants, a step size of 5 , and a pairwise $r^{2}$ threshold of 0.5 (-indep-pairwise 100050.5 ). The principal component analysis (PCA) was carried out using the GCTA 1.91 software [21]. The neighbor-joining tree [22] was constructed using PHYLIP 3.68 (evolution.genetics.washington.edu/phylip.html). MEGA7 software [23] was used to visualize the phylogenetic trees. The population structure was examined via calculating Cross Value with an expectation maximization algorithm implemented in the software ADMIXTURE [24]. The number of assumed genetic clusters $\mathrm{K}$ ranged from 2 to 7 . The population-level admixture analysis was conducted by TreeMix v.1.12 [25]. The program inferred the ML tree for 15 goat breeds (117 individuals) and an outgroup (wild goat). The command was '-l input -bootstrap -k 10000 -root outgroup -o output'. From one to 6 migration events were gradually added to the ML tree, and the command was '-i input -bootstrap -k 10000 -m migration events -o output'.

\section{Selective sweeps}

We scanned the cashmere goat genome for signatures of positive selection by combing three selection signature tests of the population-differentiation statistic $\left(F_{\mathrm{ST}}\right)$ [26], the relative nucleotide diversity $\left(\theta_{\pi}\right.$ ratio, $\left.\theta_{\pi-N o n c a s h} / \theta_{\pi-C a s h}\right)$ [27] and the transformed heterozygosity score (ZHp). Genomic evidence for positive selection in response to cashmere growth was evaluated by contrasting differentiation indices between the cashmere goats versus the other goats. $F_{\mathrm{ST}}$ and nucleotide diversity $\left(\theta_{\pi}\right)$ were calculated by VCFTools [28]. The window-based ZHp approach was calculated as previously described [29]. Each test was based on a $100-\mathrm{kb}$ window with 10-kb increment. We considered top $1 \%$ level for empirical percentile $\left(F_{\mathrm{ST}}>0.153, \theta_{\pi}\right.$ ratio $\left.>1.547,|\mathrm{ZHp}|>3.210\right)$ windows as candidate outliers in strong selective sweeps. To annotation candidate genes harbored in these selective regions, we used Rscript to map genes in selective windows. The overlapping windows shared by top $5 \%$ highest all three tests were considered as conservative candidate selection targets and were further annotated by the genomic database BioMart (http://www.biomart.org/). To detect the genomic loci that are associated with cashmere length around the $F G F 5$ gene, we also calculated the $\theta_{\pi}$ ratios, Tajima' $D$ [30] and pairwise $F_{\mathrm{ST}}$ in $2 \mathrm{Mb}$ windows between the cashmere breeds and the non-cashmere breeds. The Gene Ontology (GO) enrichment analysis of the annotated candidates were performed by using both the online G:profiler. 


\section{Validation in the extended population}

In order to predict functional candidates, this $505 \mathrm{bp}$ indel variant returning the most significant signature was classified according to their evolutionary conservation scores among other mammals. Primers were designed according to the indel region of FGF5 gene: FGF5-indel-F: 5'-GGTGATAAGCCACACGTTCAAA-3', FGF5-indel-R: 5'-TGGCTGTGATCAAACTTACAACC -3'. The indel region was genotyped by PCR amplification using the reaction condition of the 5 -min pre-denaturation, 30s-denaturation, 30s-annealing and 45 s-extension for 40 cycles. The genotype results were visualized by agarose gel electrophoresis. The indel of $F G F 5$ gene were successfully genotyped in the extended population of 288 goats, including 153 cashmere goats and 135 non-cashmere goats.

\section{Electrophoretic mobility shift assay}

The crude nuclear protein was extracted from NIH/3T3 cells using the Nuclear/Cytoplasmic Protein Extraction Kit (SINP001, Viagene) and protein concentration was determined by the Enhanced BCA Protein Assay Kit (CHEM001, Viagene). The oligonucleotide probe of the wild allele was 5'ATGACTCTGAGTCAGTCTCCTCC-3', while the oligonucleotide probe of the mutant allele was 5'ATGACTCGTCTCCTCC-3'. The probes were synthesized by Viagene Biotech company. EMSA was performed using a non-radioactive EMSA kit (SIDET101, Viagene) with biotin-probes, according to the user's manual instruction. Briefly, $4 \mu \mathrm{g}$ nuclear protein was incubated with poly dl:dC for $20 \mathrm{~min}$ at room temperature in binding reaction buffer. Then biotin-probe was added to and incubated with the mixture at room temperature for at least $20 \mathrm{~min}$. The reaction mixtures were separated by electrophoresis by $8 \%$ non-denaturing polyacrylamide gel in $0.5 \times$ Tris-borate-EDTA buffer at $120 \mathrm{~V}$ for $1 \mathrm{~h}$. The gel was transferred onto a pre-soaked nylon-membrane at $390 \mathrm{~mA}$ for $40 \mathrm{~min}$ and afterward, the energy of $800 \mathrm{~mJ}$ is applied for crosslinking DNA to nylon membrane using CL-1000 Ultraviolet Crosslinker (UVP, UK). Finally, the complexes bands were visualized by chemiluminescent detection. Competition reaction with a 80 -fold and 160-fold molar excess of unlabeled oligonucleotide were performed to confirm the specificity of the DNA-protein complex. For the supershift experiment, $2 \mu \mathrm{g}$ of anti-c-fos antibodies (sc-8047X, Santa Cruz) were added to the mixture.

\section{Dual-luciferase reporter assay}

$\mathrm{NIH} / 3 \mathrm{~T} 3$ cells were propagated in the medium of Roswell Park Memorial Institute 1640 (RPMI 1640), supplemented with $10 \%$ heat-inactivated fetal bovine serum and penicillin $(0.2 \mathrm{U} / \mathrm{ml}) /$ streptomycin $(0.2$ $\mu \mathrm{g} / \mathrm{ml}) / \mathrm{L}$-glutamine $(0.2 \mu \mathrm{g} / \mathrm{ml})$ (Gibco, USA). The 772-bp and 267-bp fragment of FGF5 indel region were cloned into the pGL4.23 vector (Promege, USA) expressing Firefly luciferase gene, respectively. We thus generated two recombinant vectors pGL4.23-ins (containing 505-bp insertion) and pGL4.23-del (containing the deletion). Each plasmid was co-transfected into NIH/3T3 cells with the internal control vector pGL4.74 expressing Renilla luciferase gene by Lipofectamine ${ }^{\mathrm{TM}} 3000$ (Invitrogen, America) according to the manufacturer's instruction. The firefly luminescence signal (FiLuc) and Renilla luciferase signal (hRLuc) of NIH/3T3 cells were measured for each transfection on a multi-function microplate 
reader (Tecan Infinite 200 Pro) using the Dual-Luciferase Reporter Assay System (E1910, Promega) after $24 \mathrm{~h}$ transfection.

\section{RT-qPCR quantification}

The total RNA was extracted from Inner Mongolia Alashan cashmere goats and Dazu black goats using RNA extraction Kit (Promega), and RNA quality and concentration were measured on an Agilent 2100 Bioanalyzer (Germany). The RIN value of samples greater than 8.0 were used for RT-qPCR analysis. The cDNA was synthesized using PrimeScript RT reagent kit with gDNA Eraser (Takara, Dalian, China) in a 20 $\mu \mathrm{l}$ reaction mixture following the manufacturer's instruction. The expression levels of FGF5 gene was normalized against UBC reference genes [31,32]. The primers used in the RT-qPCR experiment were showed in Table S11. The RT-qPCR was performed using TB Green Premix Ex Taq (Takara, Dalian, China). The qPCR reaction program was set as follow: $95^{\circ} \mathrm{C} 30 \mathrm{~s}, 40$ cycles of $5 \mathrm{~s}$ at $95^{\circ} \mathrm{C}$ and $34 \mathrm{~s}$ at $60{ }^{\circ} \mathrm{C}$. The qPCRs were run of both technical and biological replicates $(n=3)$ using an $A B I 7500$ sequence detection system (Applied Biosystems by Life Technologies, Darmstadt, Germany). Fold expression changes were determined using a standard $2^{-\Delta \Delta C T}$ method that compares $C_{T}$ (cycle threshold) values of a reference gene to the gene of interest for the $\Delta \mathrm{C}_{\mathrm{T}}$ calculation and compares the $\Delta \mathrm{C}_{\mathrm{T}}$ value of a reference sample with the sample of interest for the $\Delta \Delta \mathrm{C}_{\mathrm{T}}$ calculation [33].

\section{Results}

\section{Genomic variants}

We used the Illumina HiSeq platform to generate whole-genome sequencing data of 115 goats sampled from China, Nepal and Pakistan (Fig. 1A). The median genome coverage achieved across the full data set was $\sim 5 \mathrm{X}$ ( $\min =2.11 \mathrm{X}, \mathrm{max}=7.78 \mathrm{X}$ ), representing $\sim 87.44 \%$ ( $\min =78.54 \%$, $\max =89.88 \%$ ) base coverage per individual genome (Table S2). The alignment ratio of reads to the genome was $93.57 \%-99.93 \%$ (Table S2). Strict read alignment and genotype calling procedures allowed us to obtain a total of $17,534,538$ single nucleotide polymorphisms (SNPs). The majority of the autosomal SNPs were located within intergenic $(11,821,678,45.847 \%)$ and intronic $(11,347,329,44.007 \%)$ regions, with only $0.858 \%(221,209)$ located in exonic regions. Approximately $9 \%$ were present in downstream or upstream gene regulatory regions. A total of 61,164 missense SNPs and 126,811 synonymous SNPs resulted in a nonsynonymous/synonymous ratio of 0.482 (Table S3).

\section{Genetic diversity}

Compared to other goats, Tibetan cashmere goats were found to show the highest genome-wide heterozygosity levels, fewer runs of homozygosity (ROHs) and the lowest linkage disequilibrium (LD) decay. However, among northern Chinese cashmere goat breeds, Liaoning cashmere goat (LiNi), Alashan cashmere goat (ALS) and Arbus cashmere goat (ABS), exhibited lower genome-wide heterozygosity, more ROHs and more LD levels, while Erlangshan cashmere goat (ELS) exhibited the opposite. This result may be related to more intensive selection breeding. Imported dairy goats and Xiangdong black (XiDo) goat 
showed similarly high levels of genetic diversity. The genome-wide heterozygosity levels, ROHs and LD decay were found to be lower in Nepalese highland goats but higher in Nepalese lowland goats.

Compared to Chinese and Nepalese goat breeds, the Pakistani goat breeds showed less level of genetic diversity, especially the Bugi Toori goat, which is likely a consequence of its inbreeding history [34] (Table

\section{S4, Figs. S1 and S2).}

\section{Phylogenetic analyses}

Principal component analysis (PCA) of 3.4 million unlinked SNPs revealed that the Chinese, Nepalese and Pakistani goats can be separately clustered by the first PCA axis. One Pakistani goat breed (BTR) was genetically more distant from other goat breeds (Fig. 1B, upper panel). Restricting the analysis to Chinese goat breeds revealed four major clusters (Fig. 1B, main panel), with the first PCA axis separating the Toggenburg (TGB) and Laoshan (LaSh) dairy goat populations, the second PCA axis separating the Tibetan cashmere goat breeds, and the third PCA axis separating the XiDo black goat from southern China (Fig. S3). PC1, PC2 and PC3 were able to explain genetic differences of $3.98 \%, 3.08 \%$ and $2.90 \%$, respectively. The admixture analysis results were largely consistent with the PCA results as well as the similar genetic makeup among the Chinese, Nepalese and Pakistani goats (Fig. S4). When $\mathrm{K}=4$, the dairy goats, Chinese goats, BTR goat and the remaining goats were genetically distinct; when $\mathrm{K}=6$ and $\mathrm{K}=7$, the Chinese native goats were divided into the XiDo goat from southern Chinese, the northern Chinese cashmere goats and the Tibetan cashmere goats.

We next investigated the ML-TreeMix tree [25] and the distance-based neighbor-joining tree [22] using Capra ibex as the outgroup among all the goats. The distance-based neighbor-joining tree displayed six clades according to location or specific goat trait, which were consistent with the PCA and admixture analysis results (Fig. 1C). The reliability of the neighbor-joining tree was estimated by 100 bootstrap pseudoreplicates. The ML-TreeMix tree without migration events $(M L=0)$ inferred from the TreeMix analysis divided the 115 goats into six clusters, which were consistent with the neighbor-joining tree results (Fig. S5A). When $M=1$ and $M=2$, the results suggested that gene exchange occurred between wild and Nepalese and Pakistani goats (Fig. S5B and S5C); when M=3, genetic materials of LiNi flowed to XiDo (S5D Fig); and when M=4-6, genes flowed from XiDo to the TGB, LaSh and Nepalese lowland (NPL) goat breeds (Fig. 1D, Fig. S5D-S5F).

\section{Genome-wide selection scans for cashmere growth}

To detect the positive selection signatures within $100 \mathrm{~kb}$ sliding windows, we next scanned the cashmere goat (including TBG, TRT, LiNi, ABS, ELS, ALS and NPH) genomes relative to non-cashmere goat (including TGB, LaSh, XiDo, NPL, BTR, KMR, PTR and TPR) genomes by using three statistical methods, namely, $F_{S T}, \theta_{\pi}$ ratio $\left(\theta_{\pi-n o n c a s h} / \theta_{\pi-c a s h}\right)$ and ZHp (Fig. 2). The top-5\% selection candidates that were common to all three statistical methods identified 982 windows, which annotated a total of 378 proteincoding genes (Tables S5-S8, Fig. S6). Enrichment analyses for Gene Ontology terms revealed that the melanocortin receptor activity $(\mathrm{GO}: 0004977, P$-value $=2.33 \mathrm{E}-06)$, response to stimulus $(\mathrm{G0}: 0050896, P$ - 
value $=4.70 \mathrm{E}-07)$, developmental process $(\mathrm{GO} 00032502, P$-value $=2.07 \mathrm{E}-06)$ and cellular metabolic process $(\mathrm{GO}: 0044237, P$-value $=1.07 \mathrm{E}-06)$ categories were significantly overrepresented (Table S9). Notably, the genome window containing the FGF5 locus was under higher selection (chromosome 6, Fig. 2). This gene is known to be involved in hair growth. Moreover, several genes under strong selection signals are plausibly related to metabolism, inflammation, melanin precipitation and high-altitude adaptation. For example, STIM1 is an endoplasmic reticulum calcium sensor involved in regulating $\mathrm{Ca} 2+$ and metabotropic glutamate receptor signaling in the

nervous system $[35,36]$. The CERT protein mediates the START pathway of ceramide transport in a nonvesicular manner and the amphiphilic cavity of the START domain is optimized for specific binding of natural ceramides [37,38]. NOP14 plays significant roles in the proliferation and migration of pancreatic cancer cells [39]. The mutations in the $S G C B$ gene can lead to a loss of functional protein and result in limb-girdle muscular dystrophy disease [40]. MYCBP2 is a member of the PHR protein family and an E3 ubiquitin ligase, and it was shown to have important functions in developmental processes, such as axon termination and synapse formation [41]. WARS2 has low enzyme activity, and inhibition of WARS2 in endothelial cells reduces angiogenesis [42,43]. MC1R and KIT genes have been implicated in human and animal hair pigmentation, reflecting a role in the development and function of melanocytes [44,45]. The $D S G 3$ gene is responsible for the high-altitude adaptation of the Tibetan goat $[46,47]$.

\section{Annotation of variants under positive selection in FGF5}

We next sought to further refine the selection targets within the FGF5 locus by using three different methods, namely, the $\theta_{\pi}$ ratio $\left(\theta_{\pi \text {-noncash }} / \theta_{\pi \text {-cash }}\right)$, Tajima's $D$ and $F_{S T}$, and detecting the read depth. We noticed a 505-bp deletion within the most significant selection region in the FGF5 gene (Fig. 3,Tables S10S13), located at position 95,454,689-95,455,189 of chromosome 6 (Fig. 4A). The deletion variant is present in cashmere goats, suggesting that it arose in an independent genetic background.

We designed primers spanning the breakpoint of the deletion to genotype the indel variant by gel electrophoresis, generating a 267-bp fragment in all cashmere goats but a 772-bp fragment in noncashmere goats (Fig. 4A). To further identify the possible functional consequences of the deletion variant, we investigated whether it showed any association with cashmere growth,extending our analysis to a more comprehensive panel of 288 goats originating from 20 populations (Table S14). The results confirmed a remarkable correlation between the frequencies of the indel variant and cashmere growth. Cashmere goat breeds showed the highest allelic frequencies of deletion $(>0.9)$, whereas non-cashmere goat breeds showed higher allelic frequencies of insertion (nearly 0.8, Fig. 4B).

Furthermore, the insertion fragment of the FGF5 locus in humans showed a high conservation score in the 100-vertebrate animals alignment (e.g., goat, mouse, cat, dog, sheep, yak and donkey); by using UCSC database, we also found that the insertion fragment contains EP300, FOS and CEBPB transcription factors among different species [48,49] (Fig. 4C). This finding indicated that the indel fragment has cisregulatory effects for FGF5 gene transcription. 


\section{Biological significance of the indel variant}

Since the indel variant contains an extremely conserved FOS transcription factor binding site, we sought to functionally verify the effect of the binding site in this variant. A pair of biotin-labeled probes were designed, namely, a wildtype probe containing TGAGTCA and a mutant probe excluding the site. After the binding reaction with nucleoprotein fractions from $\mathrm{NIH} / 3 \mathrm{~T} 3$ cells derived from mouse embryonic fibroblasts, the wildtype probe resulted in an obvious positive protein complex band but the mutant probe did not. The addition of either 80 -fold or 160 -fold cold probes to the reaction system produced a significantly thinner binding band and significantly weakened grayscale compared to those with wildtype probe. The weaker binding reaction may be due to the lower cold probe concentration or higher nucleoprotein concentration making the competitive reaction incomplete. After adding c-FOS antibody to the reaction system, a complex was obviously retained at the top of the gel (Fig. 5A). We thus hypothesized that the indel variant can specifically bind to the FOS transcription factor, which may have important regulatory effects on upstream target genes.

To confirm our hypothesis, we constructed dual-luciferase recombinant plasmids either including (pGL4.23-ins) or excluding (pGL4.23-del) the indel fragment. These two recombinant vector plasmids and an empty vector were each transfected into $\mathrm{NIH} / 3 \mathrm{~T} 3$ cells together with an internal luciferase control (pGL4.74) to measure the luciferase activities. We observed significantly higher luciferase activity in cells expressing the indel fragment than both empty cells and cells expressing pGL4.23-del (Fig. 5B). This assay suggested that the indel fragment of the FGF5 gene functions as an enhancer to which certain transcription factors specifically bind to upregulate $F G F 5$ gene expression.

We next examined whether the indel mutation could alter the transcriptional response to cashmere length using RT-qPCR assays. The mRNA expression level of cashmere goats carrying the deletion was significantly decreased compared to that of non-cashmere goats carrying the insertion $(P<0.01$, Fig. $5 \mathrm{C})$. Thus, the results of the gel shift experiment, dual-luciferase assay and RT-qPCR assays confirmed that the deletion variant disrupts the binding of transcription factors (e.g. FOS) and leads to lower expression of the FGF5 gene in the skin of cashmere goats, while the insertion variant serves as an enhancer element that amplifies the transcriptional activity mediated by FGF5 in non-cashmere goats.

\section{Discussion}

In this study, we sequenced the genomes of 115 goats from 15 breeds originating from China, Nepal and Pakistan. The genome data set allowed us to identify a total of $~ 17.5$ million SNP variants, which helped us reveal the genetic diversity and population structure of these goats. In this study, most of goat breeds showed a higher diversity than others, such as the Tibetan cashmere goats, dairy goats, Erlangshan cashmere goat and Xiangdong black goat. However, the Liaoning, Alashan and Arbus cashmere goats showed lower diversity than other Chinese goats, in line with previous work [10]. The three cashmere goat breeds are famous worldwide for their fine, long fibres. This fact indicated that these breeds may have been subject to stronger intensive selection for cashmere growth. An interspecies comparison showed 
that Pakistani goats had a lower diversity than others. Notably, similar to previous work based on the goat 50K SNP chip [34], the Bugi Toori (BTR) goat breed showed the lowest genetic diversity and a great differentiation from other Pakistani goat breeds. There may have been a historical bottleneck in history or an in-flight phenomenon in the BTR goat breed (Figs. S1 and S2, Table S3).

Population structure analysis revealed that all the goats evaluated in this study were divided into six clusters, namely, dairy goat, southern Chinese goat, northern Chinese goat, Tibetan goat, Nepalese goat and Pakistani goat. When potential migration edges were added to the ML-TreeMix tree, gene exchange between the wild goats and Nepalese goats as well as Pakistani goats was detected among the clusters. This result may indicate that the local goats had a hybridization event with wild goats in the past. However, we observed migration edges between the Xiangdong black goat and Liaoning cashmere goat, as well as the dairy goat and Nepalese goat. There is a lower possibility of genetic admixture or introgresssion between the breeds because of the geographically distance between their inhabited regions. Therefore, to determine whether the southern Chinese goats underwent gene exchange with northern Chinese goat or Nepalese goats, the inclusion of more southern Chinese goat breeds is required.

(Fig. 1B-1D,Figs S3-S5).

Compared with other domestic animals, goats are more adaptable to extreme environments. In China, cashmere goats are mainly distributed in the northern China and the Tibetan Plateau, where they have adapted well to the cold environment. More importantly, the fluff produced by cashmere goats provides good, warm materials for the native population. Therefore, different cashmere traits are continuously formed under natural and artificial selection. This study compared the genomes of cashmere goats including those from habitats in Liaoning, Inner Mongolia, Tibetan areas and Nepalese highland areas bordering the Tibetan region with various non-cashmere goats from different areas. Scanning the genome of cashmere goat breeds for signatures of positive selection revealed the FGF5 gene among the top candidates (Fig. 2). The FGF5 gene participates in the FGF pathway, which plays a central role in hair growth. Studies on the FGF5 gene demonstrated the relation to coat hair length in mice [50], dogs [51], cats [52], humans [53], donkeys [54] and alpacas [55]. The same selection target has been described in a number of cashmere goat investigations $[10,56]$. In addition, disruption of the FGF5 gene via the CRISPR/Cas 9 system in cashmere goats increased the number of secondary hair follicles and enhanced the fiber length [57]. Previous studies have found a few SNP variants of the FGF5 gene that may be associated with hair length, including a missense SNP (c.284G > T) in dogs, four SNPs (c.194C>A, c.182T >A, c.474delT and c.475A>C) in cats, two SNPs (c.433_434delAT and c.245G > A) in donkeys and a missense SNP (c.499C>T) in alpacas [51,52,54,55]. Recently, one SNP (c.253G>A) in the 5'-UTR of FGF5 resulted in a start codon that could lead to a premature/dysfunctional protein in Tibetan cashmere goats [56].

At the molecular level, our work did not reveal any missense SNPs in the exons of FGF5 but instead revealed a significant indel variant in the region downstream of the FGF5 gene locus (Fig. 3). Interestingly, the result of the expanded population verification showed that the 505-bp indel variant was significantly separated in cashmere goats versus non-cashmere goats. The cashmere goats mainly exhibited a 
deletion mutation (> 0.9), whereas non-cashmere goats mainly exhibited an insertion mutation $(\sim 0.8)$. Some of the goat breeds have a small number of hybrids, such as the Nepalese lowland goat, Xiangdong black goat and Laoshan dairy goat, which may be caused by crossbreeding or altitude factors. Therefore, this result indicated that the indel variant can serve as a genetic marker for the cashmere growth trait. Furthermore, the indel mutation was found to contain a conserved binding site for the FOS transcription factor located in the mutation array and to be highly conserved in various mammals (Fig. 4). In humans, the mutation is located downstream from the FGF5 locus and has been identified as an enhancers according to the FANTOM5 Human Enhancers database (http://slidebase.binf.ku.dk/human_enhancers/). Therefore, it is speculated that the indel variant plays a potential enhancer role in FGF5 gene transcription.

Thus, an electrophoretic mobility shift assay (EMSA), and a novel dual-luciferase reporter assay based on the expression of firefly and Renilla luciferase and mRNA expression levels of FGF5 in goats were performed to explore the relevance of the indel variant to the FGF5 gene. EMSA is a powerful tool for evaluating DNA-protein or RNA-protein interaction and is often used to detected the activated transcription factors (TF) that bind with DNA or RNA in the nucleus [58]. EMSAs based on NIH/3T3 nuclear extracts revealed that the protein complex bound to the biotin-probe containing the wildtype FOS binding site, but did not bind to the probe that contained the mutant FOS binding site. Efficient competition for protein complex formation was observed with the inclusion of a wildtype cold probe, and a clear supershift occurred when anti-c-FOS antibody was added, which further confirmed that the FOS transcription factor can specifically bind to the indel variant. The c-FOS protein is a member of the FOS protein family [59]. The dual-luciferase reporter gene assay is widely used to study promoter activity, transcription factors, intracellular signaling, protein interactions [60], miRNA regulation [61], and target site recognition [62]. The dual reporter gene assay based on firefly (Photinus pyralis) and sea kidney (Renilla reniformis, also known as marine pansy) luciferases can improve experimental accuracy by normalizing results and reducing technical differences [63]. The results of this assay showed that the insertion mutation can significantly enhance promoter transcription and increase gene expression thereby verifying its enhancer function. Finally, we detected significant differences in the expression levels of the FGF5 gene from skin tissues of cashmere goats compared with non-cashmere goat, further confirming that the insertion variant may serve as an enhancer by binding to a transcription factor to result in increased transcription of its upstream FGF5 gene target (Fig. 5).

\section{Conclusions}

Our study provides a whole-genome sequence analysis of Chinese, Nepalese and Pakistani goat breeds. It includes a total of 115 individual genomes spread across 15 goat breeds. The phylogenetic relationship of the 115 individuals revealed genetically distinct clusters according to their geographic locations, but genetic admixture or introgression may have occurred between Chinese and Nepalese goats. Genomic regions showing signatures of positive selection in cashmere goats revealed that the FGF5 gene was the top candidate for the cashmere growth trait. Genotyping data from a large panel of 288 cashmere and non-cashmere goats revealed that a 505-bp indel variant, located downstream from the FGF5 gene, is 
strongly associated with the cashmere length phenotype; furthermore, the deletion fragment reached close-to-fixation ( 90\%) frequencies in cashmere goats. Functional assays demonstrated that the insertion variant may act as an enhancer by binding to transcription factor, ultimately causing increased transcription of the upstream FGF5 gene target. Our study provides useful information for the sustainable utilization and improved conservation of goat genetic resources. The valuable genetic marker that we identified will contribute to cashmere goat breeding to improve cashmere growth in the future.

\section{Abbreviations}

DNA: Deoxyribonucleic Acid

EDTA: Ethylenediaminetetraacetic acid

EMSA: Electrophoretic mobility shift assay

GO: Gene Ontology

LD: Linkage disequilibrium

PCA: Principal component analysis

PCR: Polymerase chain reaction

PHFs: Primary hair follicles

ROH: Runs of homozygosity

RT-qPCR: Quantitative reverse transcription PCR

SHFs: Secondary hair follicles

SNP: Single-nucleotide polymorphism

UTRs: Untranslated region

\section{Declarations}

Ethics approval and consent to participate

All sample collection were approved by the Animal Welfare and ethics Committee of Institute of Animal Science, Chinese Academy of Agricultural Sciences (Approval number: IAS2019-61).

\section{Consent for publication}

All authors read and approved the final manuscript. 
Availability of data and materials

The variation data reported in this paper have been deposited in the Genome Variation

Map (GVM) in Big Data Center, Beijing Institute of Genomics (BIG), Chinese Academy of Science, under accession numbers GVM000080 that are publicly accessible at

http://bigd.big.ac.cn/gvm/getProjectDetail?project=GVM000080.

\section{Competing interests}

The authors declare that they have no competing interests.

\section{Funding}

The project design and genome data generation were supported by the National Natural Science Foundation of China (Nos. 31961143021 and 31601910). Y.M. was supported by the earmarked fund for Modern Agro-industry Technology Research System (CARS-39-01) and L.J. was supported by the Elite Youth Program in Chinese Academy of Agricultural Sciences. The bioinformatics analysis and functional validation was supported by the Agricultural Science and Technology Innovation Program of China (ASTIP-IAS01).

\section{Authors' contributions}

Y.M. and L.J. conceived the project and designed the research. Y.L., S.S., L.J., Y.M., X.H., Q.Z., and Y.P. collected and prepared all the goat samples. Y.L. and S.S. performed the genetic diversity analysis. Y.L., S.S., X.L., Y.Z.,and D.W. performed the functional assays. W.G. contributed the cell cultivation. Y.L. and S.S. analyzed the bioinformatics data and validation results. Y.L., L.J. and Y.M. wrote the paper.

\section{Acknowledgments}

The authors are grateful to all goat owners and breeding organizations who donated samples. We thank members of the Nextgen project for sharing their data. We thank the National Germplasm Center of Domestic Animal Resources of IAS.

\section{References}

1. Britannica E. cashmere: Encyclopædia Britannica. 2008.

2. Von Bergen W. What the manufacturer requires in raw wool. 1963.

3. China National Bureau of Statistics. China statistical yearbook 2019. China Statistics Press. 2019.

4. Khan MS, Rehman ZU, Khan MA, Ahmad S. Genetic resources and diversity in Pakistani goats. Pak. Vet. J. 2008; 28.

5. Porter V. Goats of the world.: Farming Press; 1996.

6. Stenn KS, Paus R. Controls of hair follicle cycling. Physiol. Rev. 2001; 81: 449-494. 
7. Ansari-Renani HR, Ebadi Z, Moradi S, Baghershah HR, Ansari-Renani MY, Ameli SH. Determination of hair follicle characteristics, density and activity of Iranian cashmere goat breeds. Small Ruminant Res. 2011; 95: 128-132.

8. Zhang B, Chang L, Lan X, Asif N, Guan F, Fu D, et al. Genome-wide definition of selective sweeps reveals molecular evidence of trait-driven domestication among elite goat (Capra species) breeds for the production of dairy, cashmere, and meat. GigaScience. 2018; 7: y105.

9. Wang X, Liu J, Zhou G, Guo J, Yan H, Niu Y, et al. Whole-genome sequencing of eight goat populations for the detection of selection signatures underlying production and adaptive traits. Sci. Rep.-UK. 2016; 6: 38932.

10. Li X, Su R, Wan W, Zhang W, Jiang H, Qiao X, et al. Identification of selection signals by large-scale whole-genome resequencing of cashmere goats. Sci. Rep.-UK. 2017; 7: 1-10.

11. Bickhart DM, Rosen BD, Koren S, Sayre BL, Hastie AR, Chan S, et al. Single-molecule sequencing and chromatin conformation capture enable de novo reference assembly of the domestic goat genome. Nat. Genet. 2017; 49: 643-650.

12. Li H, Durbin R. Fast and accurate short read alignment with Burrows-Wheeler transform. Bioinformatics. 2009; 25: 1754-1760.

13. Li H. Aligning sequence reads, clone sequences and assembly contigs with BWA-MEM. arXiv preprint arXiv:1303.3997. 2013.

14. Li H, Handsaker B, Wysoker A, Fennell T, Ruan J, Homer N, et al. The sequence alignment/map format and SAMtools. Bioinformatics. 2009; 25: 2078-2079.

15. McCormick RF, Truong SK, Mullet JE. RIG: recalibration and interrelation of genomic sequence data with the GATK. G3: Genes, Genomes, Genetics. 2015; 5: 655-665.

16. Zhou Z, Jiang Y, Wang Z, Gou Z, Lyu J, Li W, et al. Resequencing 302 wild and cultivated accessions identifies genes related to domestication and improvement in soybean. Nat. Biotechnol. 2015; 33: 408-414.

17. Browning SR, Browning BL. Rapid and accurate haplotype phasing and missing-data inference for whole-genome association studies by use of localized haplotype clustering. The American Journal of Human Genetics. 2007; 81: 1084-1097.

18. Browning BL, Browning SR. Genotype imputation with millions of reference samples. The American Journal of Human Genetics. 2016; 98: 116-126.

19. Cingolani P, Platts A, Wang LL, Coon M, Nguyen T, Wang L, et al. A program for annotating and predicting the effects of single nucleotide polymorphisms, SnpEff. Fly. 2012; 6: 80-92.

20. Chang CC, Chow CC, CAM TL, Shashaank V, Purcell SM, Lee JJ. Second-generation PLINK: rising to the challenge of larger and richer datasets. Gigascience. 2015: 1.

21. Yang J, Lee SH, Goddard ME, Visscher PM. GCTA: a tool for genome-wide complex trait analysis. Am. J. Hum. Genet. 2011; 88: 76-82. 
22. SAITOU N. The neighbor-joining methods: A new method for reconstructing phylogenetic trees. Mol.biol.evol. 1987; 4.

23. Kumar S, Stecher G, Tamura K. MEGA7: molecular evolutionary genetics analysis version 7.0 for bigger datasets. Mol. Biol. Evol. 2016; 33: 1870-1874.

24. Alexander $\mathrm{DH}$, Novembre J, Lange K. Fast model-based estimation of ancestry in unrelated individuals. Genome Res. 2009.

25. Pickrell JK, Pritchard JK. Inference of population splits and mixtures from genome-wide allele frequency data. PLoS Genet. 2012; 8: e1002967.

26. Alexander DH, Novembre J, Lange K. Fast model-based estimation of ancestry in unrelated individuals. Genome Res. 2009; 19: 1655-1664.

27. Nei M, Li WH. Mathematical model for studying genetic variation in terms of restriction endonucleases. Proc Natl Acad Sci U S A. 1979; 76: 5269-5273.

28. Danecek P, Auton A, Abecasis G, Albers CA, Banks E, Depristo MA, et al. The variant call format and VCFtools. Bioinformatics. 2011; 27: 2156-2158.

29. Rubin CJ, Zody MC, Eriksson J, Meadows JR, Sherwood E, Webster MT, et al. Whole-genome resequencing reveals loci under selection during chicken domestication. Nature. 2010; 464: 587-591.

30. Tajima F. Statistical method for testing the neutral mutation hypothesis by DNA polymorphism. Genetics. 1989; 123: 585-595.

31. Bai WL, Yin RH, Yin RL, Jiang WQ, Wang JJ, Wang ZY, et al. Selection and validation of suitable reference genes in skin tissue of Liaoning cashmere goat during hair follicle cycle. Livest. Sci. 2014; 161: 28-35.

32. He X, Chao Y, Zhou G, Chen Y. Fibroblast growth factor 5-short (FGF5s) inhibits the activity of FGF5 in primary and secondary hair follicle dermal papilla cells of cashmere goats. Gene. 2016; 575: 393398.

33. Livak KJ, Schmittgen TD. Analysis of relative gene expression data using real-time quantitative PCR and the 2(-Delta Delta C(T)) Method. Methods. 2001; 25: 402.

34. Kumar C, Song S, Dewani P, Kumar M, Parkash O, Ma Y, et al. Population structure, genetic diversity and selection signatures within seven indigenous Pakistani goat populations. Anim. Genet. 2018; 49: 592-604.

35. Hartmann J, Karl RM, Alexander RP, Adelsberger H, Brill MS, Rühlmann C, et al. STIM1 controls neuronal $\mathrm{Ca}^{2} \rrbracket$ signaling, mGluR1-dependent synaptic transmission, and cerebellar motor behavior. Neuron. 2014; 82: 635-644.

36. Majewski $Ł$, Maciąg F, Boguszewski PM, Wasilewska I, Wiera G, Wójtowicz T, et al. Overexpression of STIM1 in neurons in mouse brain improves contextual learning and impairs long-term depression. Biochim Biophys Acta Mol Cell Res. 2017; 1864: 1071-1087.

37. Kudo N, Kumagai K, Tomishige N, Yamaji T, Wakatsuki S, Nishijima M, et al. Structural basis for specific lipid recognition by CERT responsible for nonvesicular trafficking of ceramide. Proc Natl 
Acad Sci U S A. 2008; 105: 488-493.

38. Bandet CL, Mahfouz R, Véret J, Sotiropoulos A, Poirier M, Giussani P, et al. Ceramide Transporter CERT Is Involved in Muscle Insulin Signaling Defects Under Lipotoxic Conditions. Diabetes. 2018; 67: 1258-1271.

39. Zhou B, Wu Q, Chen G, Zhang TP, Zhao YP. NOP14 promotes proliferation and metastasis of pancreatic cancer cells. Cancer Lett. 2012; 322: 195-203.

40. Giugliano T, Fanin M, Savarese M, Piluso G, Angelini C, Nigro V. Identification of an intragenic deletion in the SGCB gene through a re-evaluation of negative next generation sequencing results. Neuromuscul Disord. 2016; 26: 367-369.

41. Pierre S, Zhang DD, Suo J, Kern K, Tarighi N, Scholich K. Myc binding protein 2 suppresses M2-like phenotypes in macrophages during zymosan-induced inflammation in mice. Eur. J. Immunol. 2018; 48: 239-249.

42. Wang M, Sips P, Khin E, Rotival M, Sun X, Ahmed R, et al. Wars2 is a determinant of angiogenesis. Nat. Commun. 2016; 7: 12061.

43. Agnew T, Goldsworthy M, Aguilar C, Morgan A, Simon M, Hilton H, et al. A Wars2 Mutant Mouse Model Displays OXPHOS Deficiencies and Activation of Tissue-Specific Stress Response Pathways. Cell Rep. 2018; 25: 3315-3328.

44. Chen S, Zhu B, Yin C, Liu W, Han C, Chen B, et al. Palmitoylation-dependent activation of MC1R prevents melanomagenesis. Nature. 2017; 549: 399-403.

45. Moss KG, Toner GC, Cherrington JM, Mendel DB, Laird AD. Hair depigmentation is a biological readout for pharmacological inhibition of KIT in mice and humans. J. Pharmacol. Exp. Ther. 2003; 307: 476-480.

46. Kumar C, Song S, Jiang L, He X, Zhao Q, Pu Y, et al. Sequence Characterization of DSG3 Gene to Know Its Role in High-Altitude Hypoxia Adaptation in the Chinese Cashmere Goat. Front Genet. 2018; 9: 553.

47. Song S, Yao N, Yang M, Liu X, Dong K, Zhao Q, et al. Exome sequencing reveals genetic differentiation due to high-altitude adaptation in the Tibetan cashmere goat (Capra hircus). BMC Genomics. 2016; 17: 122.

48. Gerstein MB, Kundaje A, Hariharan M, Landt SG, Yan K, Cheng C, et al. Architecture of the human regulatory network derived from ENCODE data. Nature. 2012; 489: 91-100.

49. Wang J, Zhuang J, lyer S, Lin X, Whitfield TW, Greven MC, et al. Sequence features and chromatin structure around the genomic regions bound by 119 human transcription factors. Genome Res. 2012; 22: 1798-1812.

50. Hébert JM, Rosenquist T, Götz J, Martin GR. FGF5 as a regulator of the hair growth cycle: evidence from targeted and spontaneous mutations. Cell. 1994; 78: 1017-1025.

51. Housley D, Venta PJ. The long and the short of it: evidence that FGF5 is a major determinant of canine 'hair'-itability. Anim. Genet. 2006; 37: 309-315. 
52. Drögemüller $\mathrm{C}$, Rüfenacht $\mathrm{S}$, Wichert $\mathrm{B}$, Leeb T. Mutations within the FGF5 gene are associated with hair length in cats. Anim. Genet. 2007; 38: 218-221.

53. Higgins CA, Petukhova L, Harel S, Ho YY, Drill E, Shapiro L, et al. FGF5 is a crucial regulator of hair length in humans. Proceedings of the National Academy of Sciences. 2014; 111: 10648-10653.

54. Legrand $\mathrm{R}$, Tiret $\mathrm{L}$, Abitbol M. Two recessive mutations in FGF5 are associated with the long-hair phenotype in donkeys. Genet. Sel. Evol. 2014; 46: 1-7.

55. Pallotti S, Pediconi D, Subramanian D, Molina MG, Antonini M, Morelli MB, et al. Evidence of posttranscriptional readthrough regulation in FGF5 gene of alpaca. Gene. 2018; 647: 121-128.

56. Guo J, Zhong J, Li L, Zhong T, Wang L, Song T, et al. Comparative genome analyses reveal the unique genetic composition and selection signals underlying the phenotypic characteristics of three Chinese domestic goat breeds. Genet. Sel. Evol. 2019; 51: 70.

57. Wang X, Cai B, Zhou J, Zhu H, Niu Y, Ma B, et al. Disruption of FGF5 in cashmere goats using CRISPR/Cas9 results in more secondary hair follicles and longer fibers. PLoS One. 2016; 11: e164640.

58. Garner MM, Revzin A. The use of gel electrophoresis to detect and study nucleic acid-protein interactions. Trends Biochem. Sci. 1986; 11: 395-396.

59. Milde-Langosch K. The Fos family of transcription factors and their role in tumourigenesis. Eur. J. Cancer. 2005; 41: 2449-2461.

60. Jia S, Peng J, Gao B, Chen Z, Zhou Y, Fu Q, et al. Relative quantification of protein-protein interactions using a dual luciferase reporter pull-down assay system. PLoS One. 2011; 6: e26414.

61. Jin Y, Chen Z, Liu X, Zhou X. Evaluating the microRNA targeting sites by luciferase reporter gene assay. Methods Mol Biol. 2013; 936: 117-127.

62. Jin Y, Chen Z, Liu X, Zhou X. Evaluating the microRNA targeting sites by luciferase reporter gene assay. MicroRNA protocols: Springer. 2013. pp. 117-127.

63. Stables J, Scott S, Brown S, Roelant C, Burns D, Lee MG, et al. Development of a dual glow-signal firefly and Renilla luciferase assay reagent for the analysis of G-protein coupled receptor signalling. J. Recept. Sig. Transd. 1999; 19: 395-410.

\section{Figures}



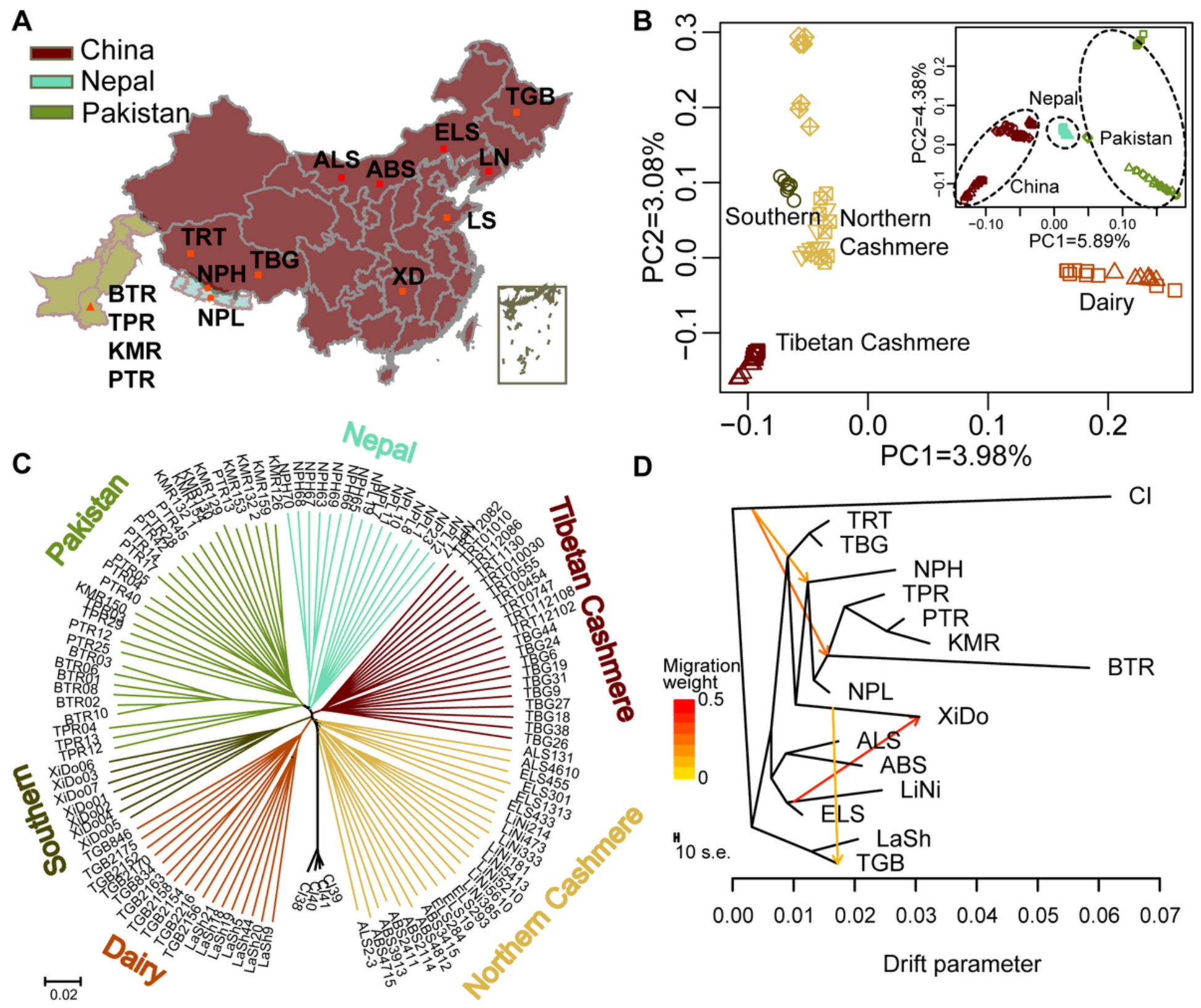

Figure 1

Geographic distribution, genetic structure of Chinese, Nepalese and Pakistani goat breeds. (A) The geographic distribution of 15 goat populations. The red color represent Chinese goats (TGB, Toggenburg dairy goat from Heilongjiang; LaSh, Laoshan dairy goat from Shandong; LiNi, Liaoning cashmere goat from Liaoning; ABS, Arbus cashmere goat; ELS, Erlangshan cashmere goat; ALS, Alashan cashmere goat from Inner Mongolia; TBG, Tibetan Bangor cashmere goat; TRT, Tibetan Ritu cashmere goat from Tibet; XiDo, Xiangdong black goat from Hunan). The blue color represents Nepalese goats (NPH, Nepalese Highland goat; NPL, Nepalese Lowand goat). The green color represents Pakistani goats (BTR, Bugi Toori goat; KMR, Kamori goat; PTR, Pateri goat; TPR, Tapri goat). (B) PCA plots of the first two components of all goats (inner plot) and Chinese goats (outer plot). The fraction of the total variance explained is reported on each individual axis between parentheses. (C) The neighbor-joining tree of the goat breeds, with Capra ibex as the outgroup. Bootstrap reported was close to $100 \%$. (D) The ML-TreeMix tree of all 
goats, with Capra ibex as the outgroup, assuming four migration events. Migration arrows are colored according to their weights. Horizontal branch lengths are proportional to the amount of genetic drift parameter that has occurred on the branch. The drift parameter measures the variance in allele frequency estimated along each branch of the tree. The yellow and orange lines indicate the instantaneous admixtures, whereas arrows denote continuous (unidirectional) genes flow.

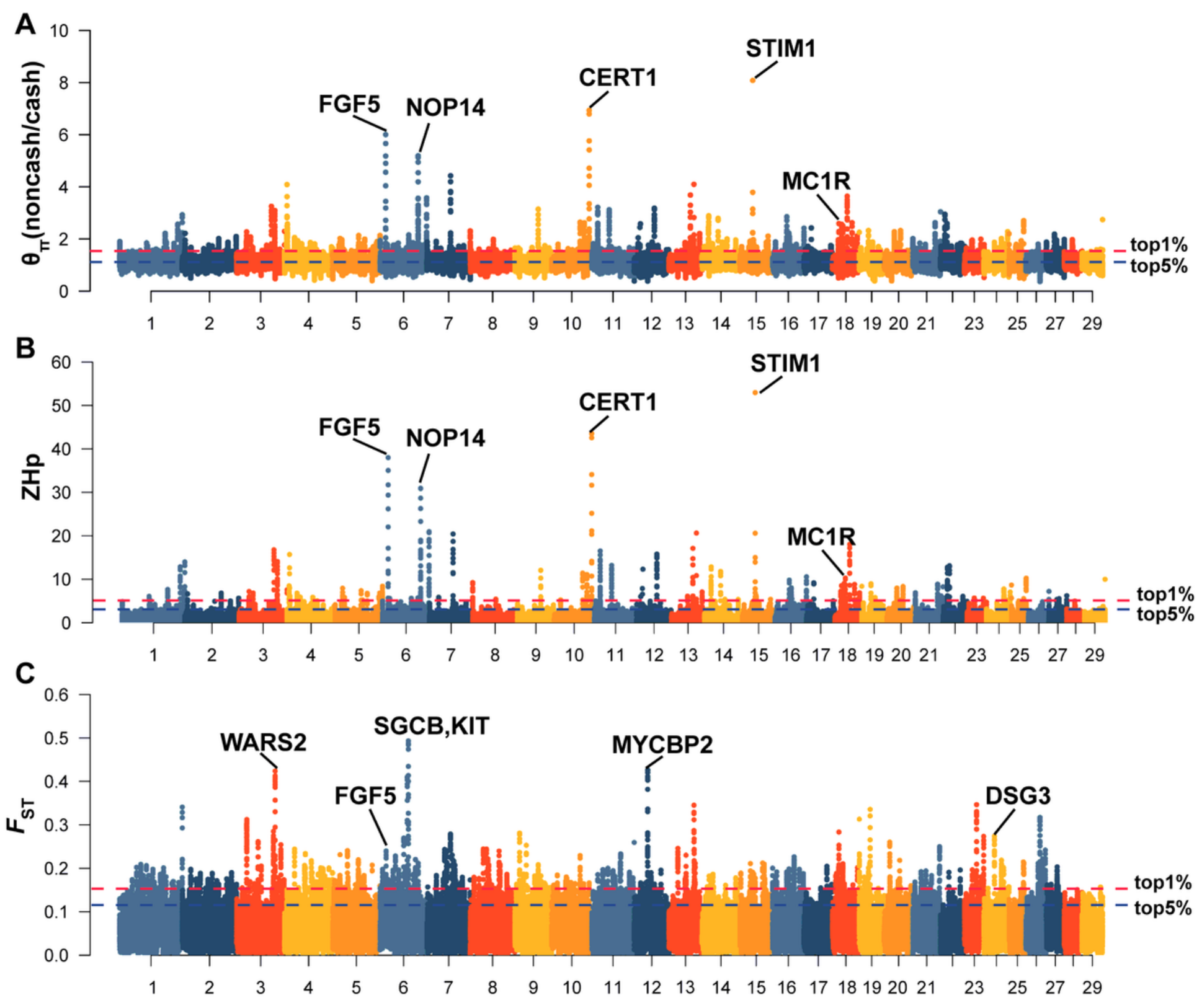

\section{Figure 2}

Positive selection scans for cashmere growth. Cashmere goats are compared with non-cashmere goats. The nucleotide diversity $\theta \pi$ ratio $(\theta \pi$-noncash/ $\theta \pi-c a s h)(A)$,the transformed heterozygosity score ZHp (B) and the population genetic differentiation FST values (C) are calculated within $100 \mathrm{~kb}$ sliding windows 
(step size $10 \mathrm{~kb}$ ). The significance threshold of a selection signature was arbitrarily set to the top $5 \%$ percentile outliers for each individual test and is indicated with blue horizontal dashed lines. The red horizontal dashed lines delineate the top $1 \%$ quantile.

A
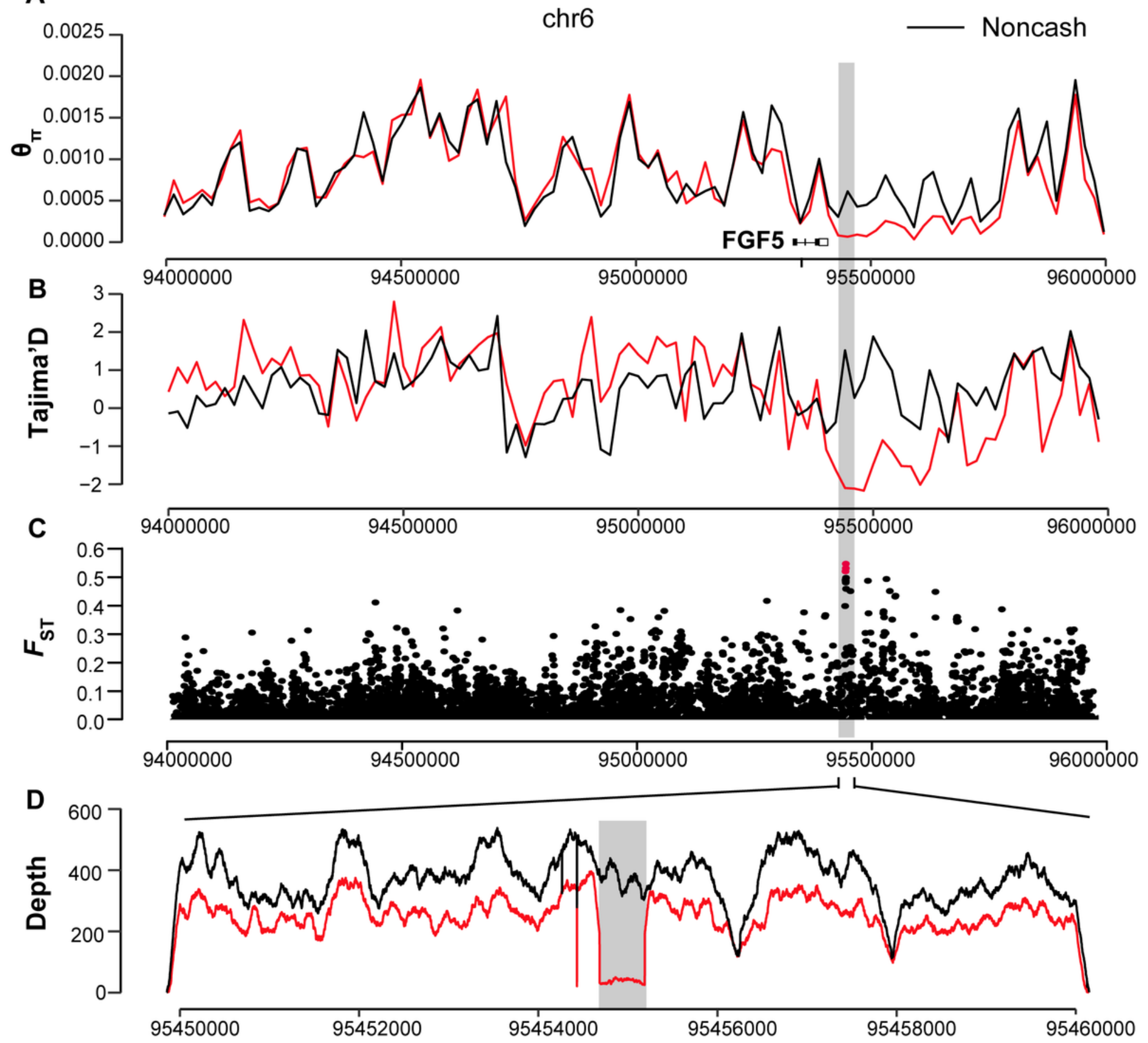

Figure 3

The strongest positive selection signature around the FGF5 peak. The $\theta \pi$ ratio (A), Tajima's D (B) and FST value (C) are plotted against the peak position from $94.0 \mathrm{Mb}$ to $96.0 \mathrm{Mb}$, and the read depth value (D) is plotted against the peak position from 95.45 Mb to $95.46 \mathrm{Mb}$ on chromosome 6 . Both $\theta \pi$ ratio and Tajima's D values were based on a $20 \mathrm{~kb}$ window and a $20 \mathrm{~kb}$ step. The red and the black lines represent cashmere and non-cashmere goats, respectively. The gray columns represent the strongest positive 
selection signature in the region considered. The small black boxes and short lines represent the gene structure of FGF5.

A

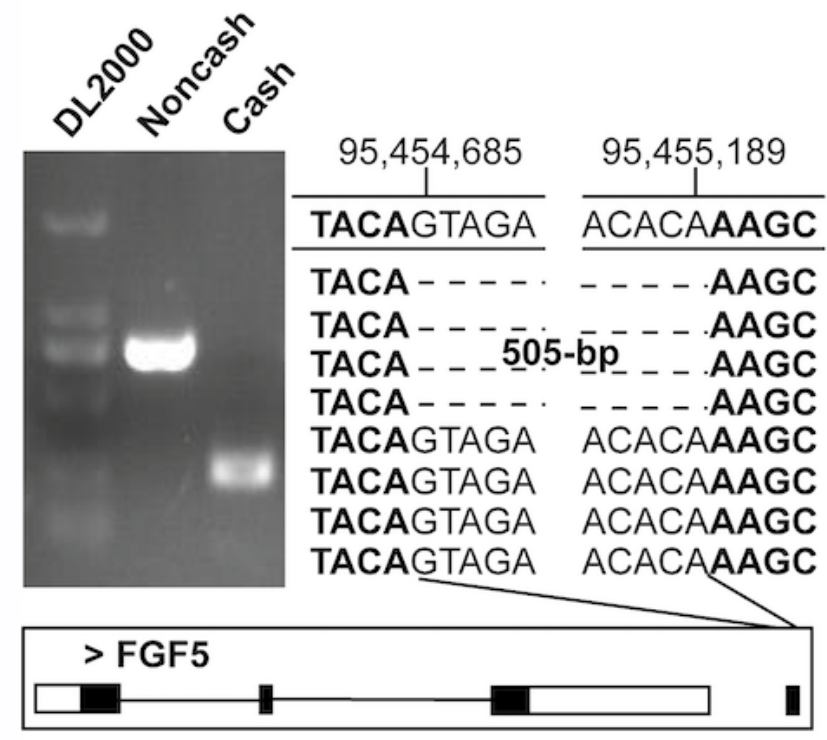

B

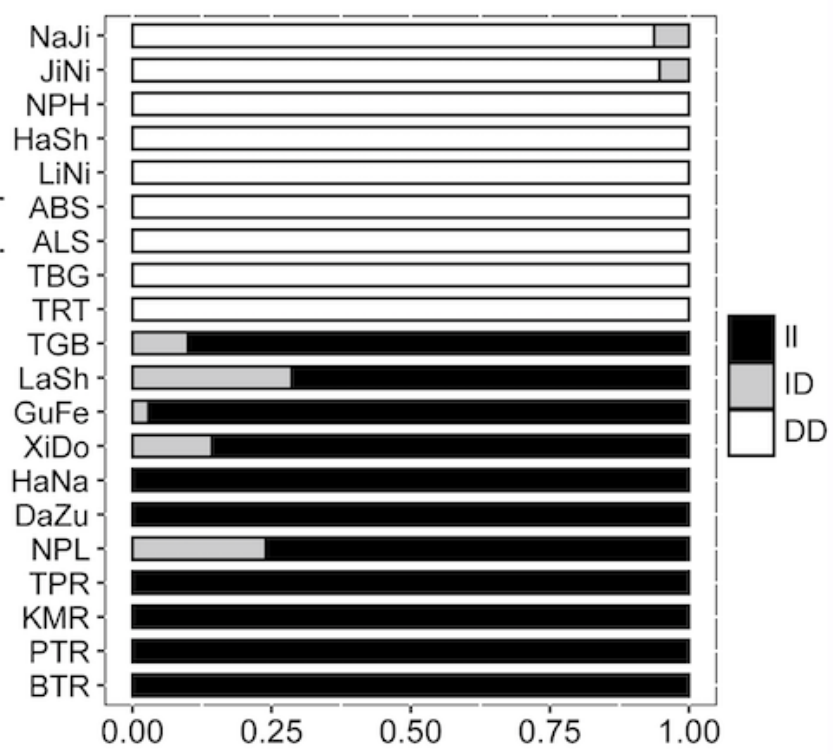

C

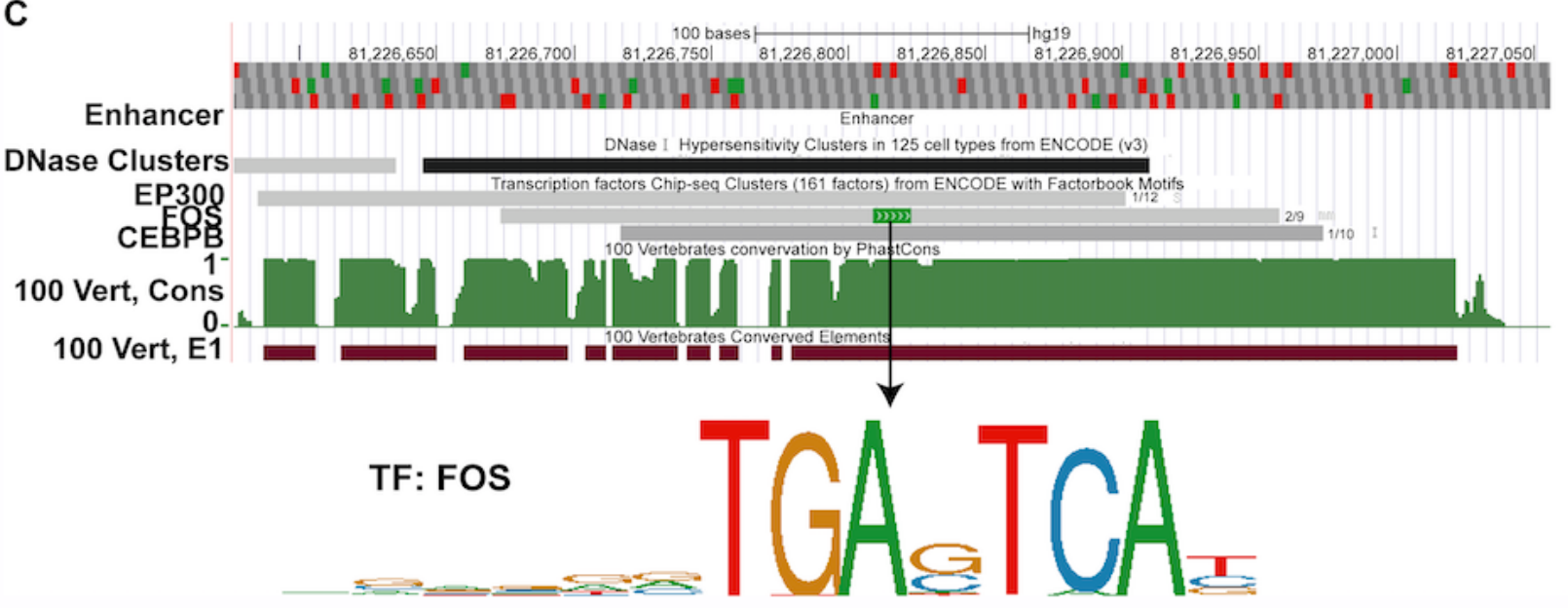

Figure 4

Annotation of the indel variant in the FGF5 gene showing positive selection signatures. (A) The PCR amplification of 505-bp indel variant, generating a 267-bp fragment in all cashmere goats while a 772-bp fragment in non-cashmere goats. The indel is located at position 95,454,689-95,455,189 of chromosome 6 in the downstream of FGF5 gene. (B) Genotypes of indel were determined in a larger population ( $N=$ 288 goats). II represents homozygous insertion genotype; ID represents heterozygous indel genotype; DD represents homozygous deletion genotype. Cashmere goat breeds include NaJi (Nanjiang cashmere goat), JiNi (Jining grey goat), NPH (Nepalese highland goat), HaSh (Hanshan white cashmere goat), LiNi 
(Liaoning cashmere goat), ABS (Arbus cashmere goat), ALS (Alashan cashmere goat), TBG (Tibetan Bangor cashmere goat) and TRT (Tibetan Ritu cashmere goat). Non-cashmere goat breeds include TGB (Toggenburg dairy goat), LaSh (Laoshan dairy goat), GuFe (Guangfeng goat), XiDo (Xiangdong black goat), HaNa (Hainan black goat), DaZu (Dazu black goat), NPL (Nepalese lowland goat), BTR (Bugi Toori goat), KMR (Kamori goat), PTR (Pateri goat) and TPR (Tapri goat). (C) The insertion fragment of FGF5 gene in humans contains a highly conserved FOS transcription factor binding site (TGAGTCA) in the UCSC database.
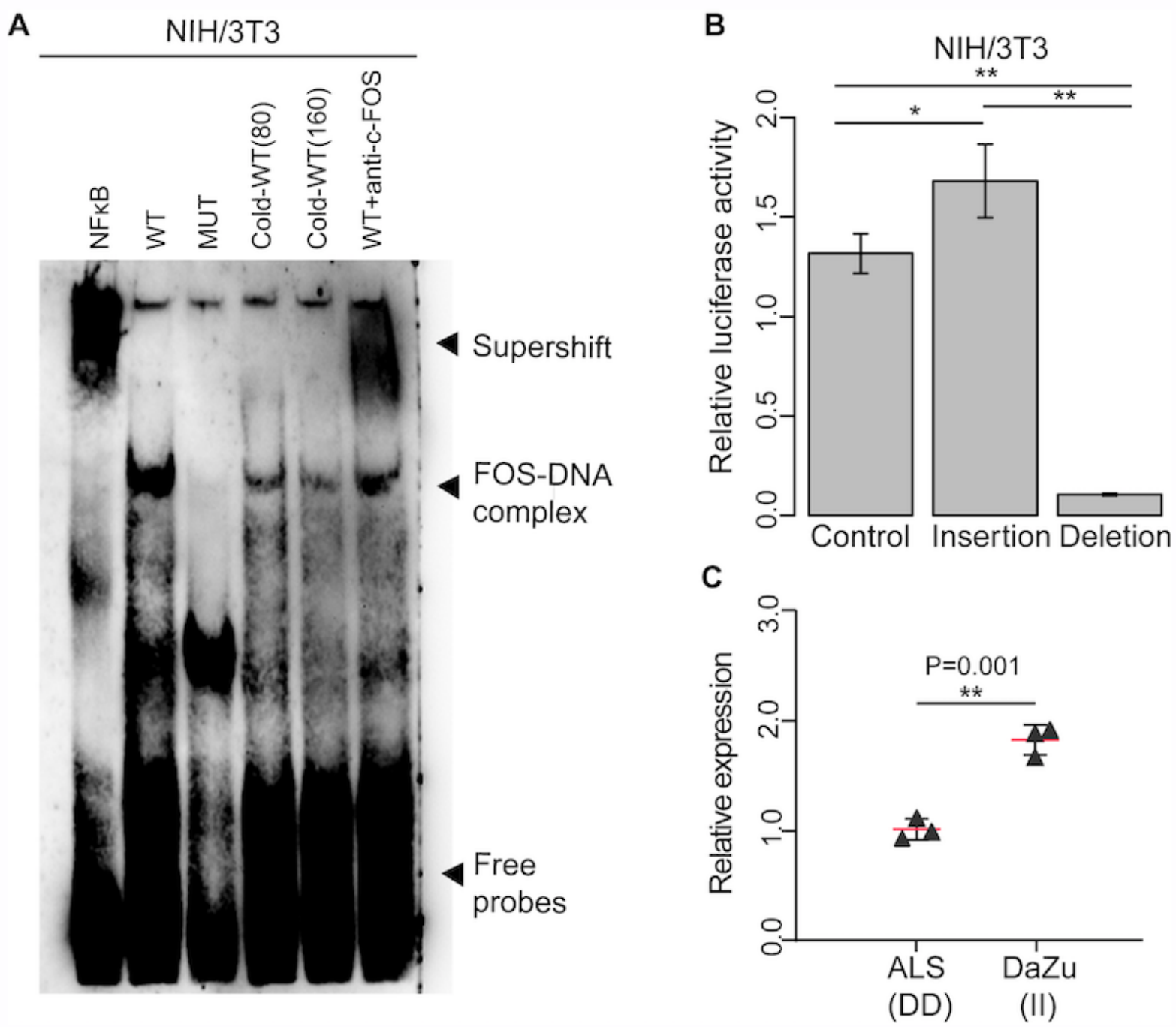

\section{Figure 5}

Validation of the indel variant in the FGF5 gene. (A) Electrophoretic mobility shift assays (EMSAs) using the nuclear protein from NIH/3T3 cells. NFKB acts as a positive control (lane 1). WT and MUT represent the probe containing the wildtype FOS binding site and the probe excluding the FOS binding site (lane 2 and 3), respectively. The cold competitions of the protein complex formation by 80 and 160 fold over that 
of wildtype probe (lane 4 and 5). The clear supershift with anti-c-FOS antibody mixtured to wildtype react (lane 6). (B) Dual-luciferase activity assay of the NIH/3T3 cell lysates cotransfected with the pGL4.74 internal reference plasmid and the pGL4.23 empty vector as control, the pGL4.23 recombinant plasmids of the insertion or the deletion variant. (C) The qPCR gene expression of the FGF5 gene in the skin of ALS (Alashan cashmere goat) and DaZu black goats (non-cashmere goat). * and ** displayed the statistical significance of P-values $<0.05$ and 0.01 , respectively.

\section{Supplementary Files}

This is a list of supplementary files associated with this preprint. Click to download.

- TablesSupplnfo.xlsx

- TablesSupplnfo.xIsx

- FiguresSupplnfo.docx

- FiguresSupplnfo.docx 